\title{
Depresión asociada al apoyo social, estancia hospitalaria y patología médica en pacientes ingresados en hospital de clínicas Dr. Manuel Quintela Depression linked to social support, hospital stay and medical pathology in patients admitted in hospital de clínicas Dr. Manuel Quintela*
}

\author{
Alejandra Moreira R. ${ }^{1}$, Pablo Fieltiz G. ${ }^{2}$, Gabriela López R. ${ }^{6}$ \\ Colaboradores: Romina Aguerre ${ }^{3}$, Guillermo Ezeiza $^{4}$, Viviana Martino ${ }^{4}$, Gilberto Correa ${ }^{5}$, \\ Sofía Decaro ${ }^{5}$, Carolina Fernández ${ }^{5}$, Silvia Gorgoroso ${ }^{5}$, Felicia Torres ${ }^{5}$, Mary Ferreira ${ }^{5}$, \\ Viviana Navarro ${ }^{6}$, Natalia Da Silva Real ${ }^{7}$ Lía Redes $^{6}$
}

\begin{abstract}
Introduction: The prevalence of depression and anxiety in pacients hospitalized in general hospitals is very high, this is associated with increased morbidity and mortality and higher health costs. Emotional support is essential in any recovery process. Method: A descriptive and analytical study was carried out on depression and its relationship with social support, hospital stay and medical illness in patients admitted to the Dr. Manuel Quintela Clinical Hospital Montevideo - Uruguay. The Beck test for depression and the scale validated for social support Medical Outcomes Study (MOS) were applied. Characteristics of the studied population were described. Results: More than half of the interviewees presented some degree of depesion. It was possible to affirm that $73.7 \%$ of the users with scarce social support presented depressive symptoms with an increase in depression, especially of moderate and mild type. There was a higher incidence of depression in patients with prolonged hospital stay, however this data was not statistically significant. Regarding medical pathology, we highlight the strong presence of depression in cardiovascular and surgical oncology patients.

Key words: Depression, social support, medical disease.
\end{abstract}

Rev Chil Neuro-Psiquiat 2018; 56 (4): 219-227

Recibido: 23/06/2018

Aprobado: 04/12/2018

Los autores no presentan ningún tipo de conflicto de interés.

*Clínica Psiquiátrica Facultad de Medicina. Montevideo, Uruguay. Octubre 2017.

Médico Psiquiatra, Asistente de Clínica Psiquátrica Facultad de Medicina, Montevideo, Uruguay.

Médico Psiquiatra, Profesor Agregado de Clínica Psiquiátrica Facultad de Medicina, Montevideo, Uruguay.

Médico Psiquiatra.

Doctor en Medicina Residente de Clínica Psiquiátrica Facultad de Medicina. Montevideo, Uruguay.

Doctor en Medicina, Postgrado de Clínica Psiquiátrica Facultad de Medicina. Montevideo, Uruguay.

Médico Psiquiatra, Profesor Adjunto de Clínica Psiquiátrica Facultad de Medicina. Montevideo, Uruguay.

Licenciado en Psicología Facultad de Psicología. Montevideo, Uruguay. 


\section{Introducción}

$\mathrm{L}$ a depresión es un trastorno primario del estado de ánimo que afecta el funcionamiento global, con sentimientos persistentes de inutilidad, infravaloración personal, pérdida de interés por el mundo y falta de esperanza en el futuro. Su etiología es multifactorial, lo que conlleva la inclusión de factores genéticos, ambientales y temperamentales ${ }^{1}$.

La prevalencia de depresión y ansiedad en hospitales generales es muy alta ${ }^{2-4}$, esto constituye un problema que afecta la salud de los usuarios y su pronóstico vital. La depresión se ha asociado a una estancia hospitalaria más prolongada, mayores costos y mayor número de procedimientos ${ }^{2}$ Los usuarios deprimidos presentan una alteración de su estado de salud, un aumento del uso del sistema de atención y mayor riesgo de no adherencia al tratamiento ${ }^{5}$. Muchas veces no se indica consulta oportuna con especialista dado que la sintomatología es atribuida al proceso de hospitalización y a la repercusión de la enfermedad orgánica.

La asociación entre enfermedad física y mental ha sido motivo de múltiples investigaciones en nuestro medio, varios de estos trabajos no han llegado a ser publicados.

En el Hospital de Clínicas según estudio publicado en 2014 la frecuencia de depresión entre los pacientes que consultan en policlínica resultó mayor a los valores de referencia para la población general $^{6}$.

Un estudio nacional realizado en el Hospital Pasteur cuantificó depresión y ansiedad en usuarios hospitalizados con una incidencia de $50 \%{ }^{7}$.

En un estudio chileno para usuarios mayores de 60 años ingresados en un Servicio de Medicina Interna la prevalencia de depresión fue de $22,13 \%$, $28,12 \%$ en mujeres y $16,41 \%$ en hombres ${ }^{8}$.

Según un estudio publicado en Buenos Aires la depresión en los hospitales metropolitanos es de un $27 \%$ cuantificada por examen clínico psiquiátrico a un $44 \%$ con inventario de Beck 9 .

En Colombia la prevalencia de depresión en hospitales de la ciudad de Bucaramanga llega al $41,5 \%$ con mayor prevalencia de síntomas somático-motivacionales, frente a los síntomas cognitivo-afectivos ${ }^{10}$. El acompañamiento de familiares y seres queridos resulta fundamental en los procesos de recuperación tanto a nivel físico como emocional. Por lo que, los programas de psicoeducación a familiares y encargados son una alternativa que se debe considerar, si se pretende promover una mejor calidad de vida para las personas hospitalizadas ${ }^{11}$.

\section{Objetivos}

Describir la población de estudio.

Evaluar las posibles asociaciones entre la presencia de síntomatología depresiva, soporte social, estancia hospitalaria y patología médica.

Implementar medidas terapéuticas específicas en los casos detectados si aún no se hubieran iniciado.

\section{Diseño y Métodos}

El estudio es de tipo descriptivo, analítico, transversal y observacional ${ }^{12,13}$.

La población se compone por el universo de pacientes internados en el Hospital de Clínicas Dr. Manuel Quintela Montevideo-Uruguay. Este es un hospital universitario que funciona como hospital general de adultos de alta complejidad, de referencia nacional ${ }^{14}$. Según datos aportados por centro de estadística del Hospital de Clínicas el promedio de camas ocupadas en 2017 fue de 138 en cuidados moderados medicina, 154 cuidados moderados cirugía, 12 cuidados intermedios, 10 en cuidados intensivos, 314 en total.

A los pacientes hospitalizados en el período 7-9 de octubre de 2015 se les aplicó la escala validada de Beck para depresión ${ }^{15}$ en su versión revisada BDI-I. Los puntos de corte son: 0-9 altibajos normales del estado de ánimo, 10-18 depresión leve a moderada, 19-29 depresión entre moderada y severa, de 30 a 63 depresión severa.

El inventario de depresión de Beck es una herramienta de gran extensión ampliamente manejada en investigación. En algunos grupos poblacionales su validez ha sido puesta en duda, por ejemplo en los pacientes con patología médica ${ }^{16}$. 
La preocupación acerca de la validez e interpretación de las escalas de depresión para distinguir los trastornos afectivos y orgánicos ha llevado a los investigadores a experimentar con nuevos procedimientos para calificarlos y/o modificar su contenido. Cavanaugh, Clark y Gibbons (1983) dividieron los ítems del Inventario de Depresión de Beck (BDI) en subescalas que miden los síntomas cognitivos/afectivos y los somático/ejecutivos. Totalizaron las respuestas de varios grupos de sujetos a cada componente del Inventario en lugar de a su totalidad, y concluyeron que la depresión en pacientes médicos se mide mejor por los elementos cognitivos/afectivos ya que están relacionados positivamente con la gravedad. Es recomendable que en pacientes somáticamente enfermos se usen solo los ítems cognitivo-afectivos ${ }^{17}$.

Según estudio peruano los ítems que mejor predicen la presencia de depresión son: la inconformidad con uno mismo, la autopercepción negativa del aspecto físico, la autocensura, el insomnio y el sentirse castigado. Concluyen que la versión en español del BDI utilizada tiene propiedades psicométricas adecuadas para la evaluación de depresión en pacientes hospitalizados de medicina general ${ }^{18}$.

También se aplicó el formulario validado Medical Outcomes Study (MOS) ${ }^{19}$, que mide el apoyo social en pacientes con enfermedades crónicas. Definimos al apoyo social escaso como un puntaje inferior a 57 en el cuestionario MOS.

Adicionalmente, se completó una ficha ${ }^{20}$, en la cual se recogieron reseñas patronímicas, información vinculada con la patología médica, fecha de ingreso al hospital.

Cruzamos la variable dependiente depresión con: apoyo social, estancia hospitalaria y enfermedad médica.

Utilizamos para el análisis estadístico el sistema Statistical Package for the Social Sciences (SSPS). Los test de significación estadística fueron empleados con el fin de trascender el análisis descriptivo (prueba U de Mann-Whitney) para las variables dicotómicas relevadas (sexo, si recibe tratamiento psiquiátrico, ideas de muerte, antecedentes psiquiátricos y apoyo social) con el fin de contrastar la diferencia entre los niveles de depresión ordinales (medidos a través del test de Beck). Por otro lado, para el análisis entre las variables "Ideas de muerte" y "Apoyo social” (MOSS), se aplicó Phi de Pearson para variables nominales.

Fueron criterios de exclusión la presencia de cualquier alteración mental que impida consentir libremente o invalide las escalas utilizadas, por ejemplo: síndrome confusional, retardo mental, demencia. También fueron excluidos todos los usuarios que no deseaban participar o su condición médica se los impedía violando el principio de autonomía. Se excluyeron los pacientes gravemente enfermos (Servicio de CTI y CENAQUE) o en aislamiento por motivos infecciosos.

\section{Puntualizaciones éticas}

Cada uno de los pacientes implicados ha autorizado la lectura y uso de datos obtenidos en entrevista clínica con fines científicos, mediante un consentimiento informado firmado válido.

Los casos de depresión detectados fueron captados y tratados, se les ofreció seguimiento en policlínica de psiquiatría del Hospital de Clínicas.

Los datos han sido tratados de manera confidencial omitiendo nombres propios respetando los derechos del paciente establecidos en la Ley $18.335^{21}$.

Los autores de este estudio no declaran ningún conflicto de intereses. No contamos con financiación.

Se acatan las normas, códigos y declaraciones internacionales de ética médica ${ }^{22-25}$.

\section{Resultados}

De un total de 170 usuarios internados, 21 no se encontraban en condiciones médicas de ser entrevistados, 19 estaban aislados, 18 no aceptaron formar parte del estudio, 16 se encontraban en interconsulta con otra especialidad o realizándose estudios. Finalmente, de los 96 pacientes entrevistados, 44 eran de sexo masculino y 52 de sexo femenino. La mayoría (61,5\%) eran mayores de 46 años. En cuanto al nivel de instrucción, el 93,8\% tenía al menos primaria completa. El 31,3\% eran 
jubilados/pensionistas y el $21,9 \%$ se encontraba desocupadas al momento de ser entrevistados. El $21,9 \%$ presentó antecedentes personales psiquiátricos.

En lo referente a los antecedentes personales médicos el $27,1 \%$ poseían polidiagnóstico, $17,7 \%$ presentaban antecedentes cardiovasculares.

El 30,2\% de los pacientes permanecieron internados menos de 5 días, $61,5 \%$ entre 6 a 40 días, $8,3 \%$ más de 40 días.

El 19,8\% tuvo como motivo de ingreso patología cardiovascular seguido de mayor a menor frecuencia por: gineco-obstétrico $15,6 \%$, neoplásico $14,6 \%$, neurológico, infeccioso y quirúrgico $11,5 \%$, osteoarticular $2,1 \%$ y otros $13,5 \%$.

Como vemos en la Figura 1 más de la mitad de los usuarios entrevistados tenían algún grado de depresión. Quienes padecían depresión leve representaban más de un tercio de los casos, $10,4 \%$ presentaban depresión moderada y $5,2 \%$ depresión severa.

El 5,2\% de los pacientes presentaron ideas de autoeliminación. El 88\% de quienes negaron estas ideas no presentaron depresión o presentaron depresión leve. El 80\% de los pacientes que declararon haber experimentado ideas de autoeliminación presentaron niveles depresivos moderados y severos. Asimismo, es preciso observar que todos los pacientes que presentaron ideas de autoeli- minación tenían algún tipo de depresión (leve, moderada, severa).

En la Figura 2 observamos que en los pacientes más gravemente deprimidos el puntaje total del test de Beck fue a predominancia de los items somato ejecutivos coincidiendo esto con lo descripto por Beck y Cols y apoyando la necesidad de dar preponderancia a la parte cognitivo afectiva del test ${ }^{17}$.

En la Figura 3 vemos que la mayor parte $(73,7 \%)$ de los usuarios con apoyo social escaso presentaron síntomas depresivos con un aumento de la incidencia de depresión sobre todo de tipo moderada y leve. Sin embargo, el porcentaje de depresión severa fue igual para ambos grupos.

Detectamos más depresión en pacientes con estancia hospitalaria prolongada, sin embargo, este dato no fue estadísticamente significativo.

En cuanto a la patología médica destacamos la fuerte presencia de depresión en los pacientes oncológicos cardiovasculares y quirúrgicos. Dentro de los pacientes que ingresaron por enfermedades de tipo neurológico se aprecia que el 36,4\% presentaron altibajos normales del ánimo. Así también, más de la mitad $(63,2 \%)$ de quienes ingresaron por problemas cardiovasculares, poseían algún nievel de depresión. Por su parte, el 36,4\% de los pacientes quirúrgicos presentó depresión leve, y el 18,2\% depresión moderada (Figura 4).

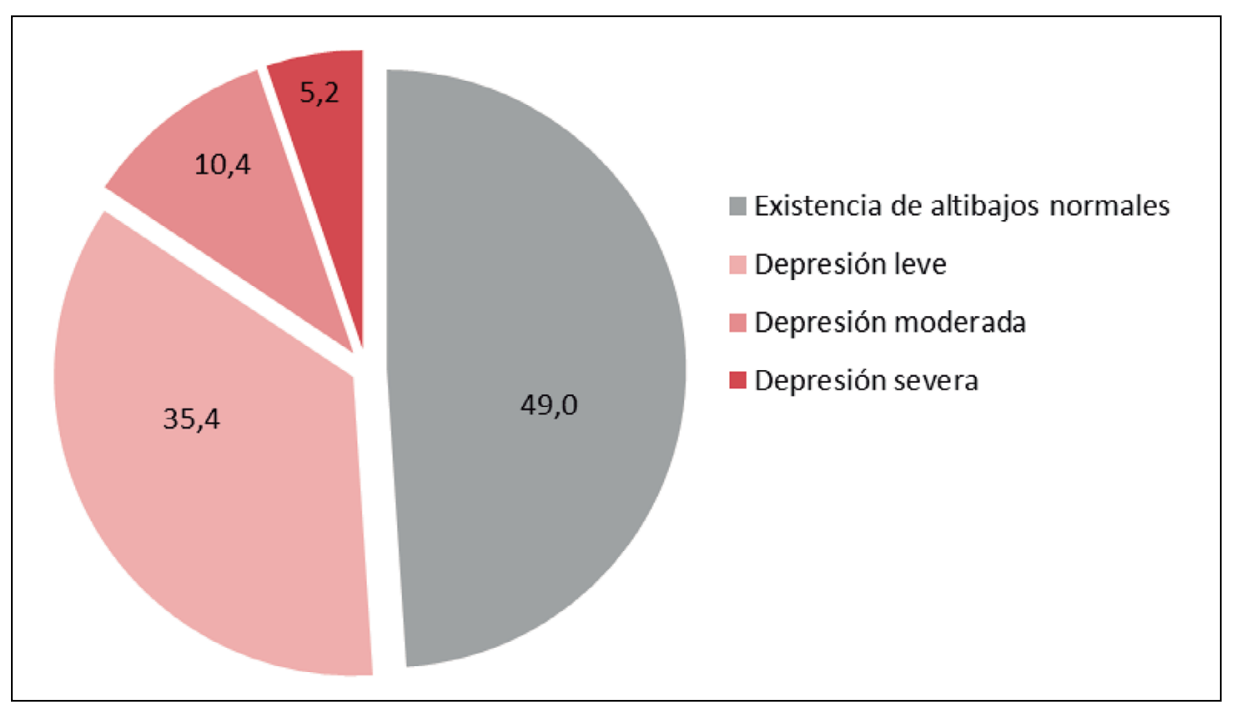

Figura 1. Prevalencia medida en porcentaje de los resultados del test de Beck. 


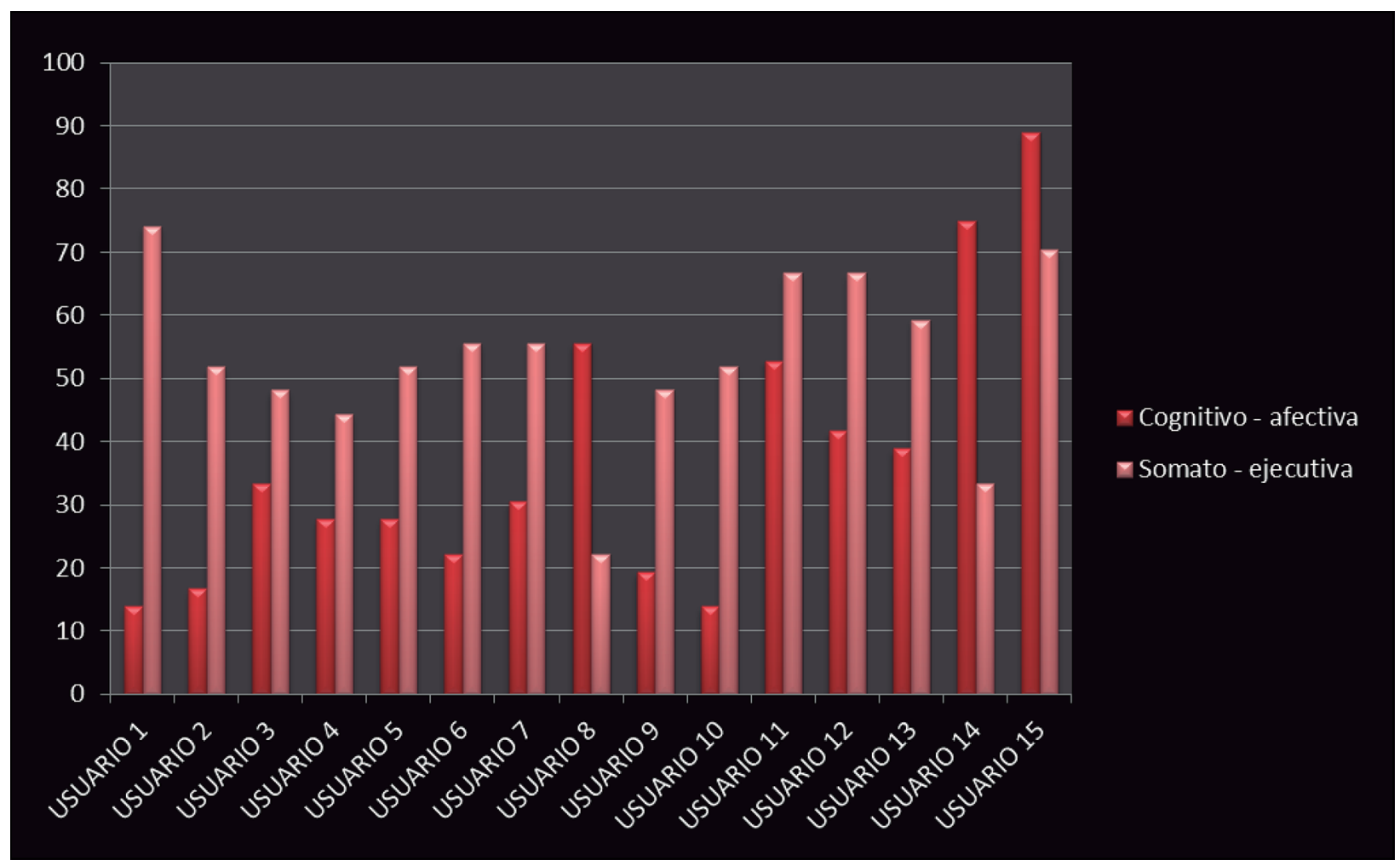

Figura 2. Discriminación de resultados de preguntas del test de Beck en cognitivo afectivas y somato ejecutivas en los pacientes más gravemente deprimidos.

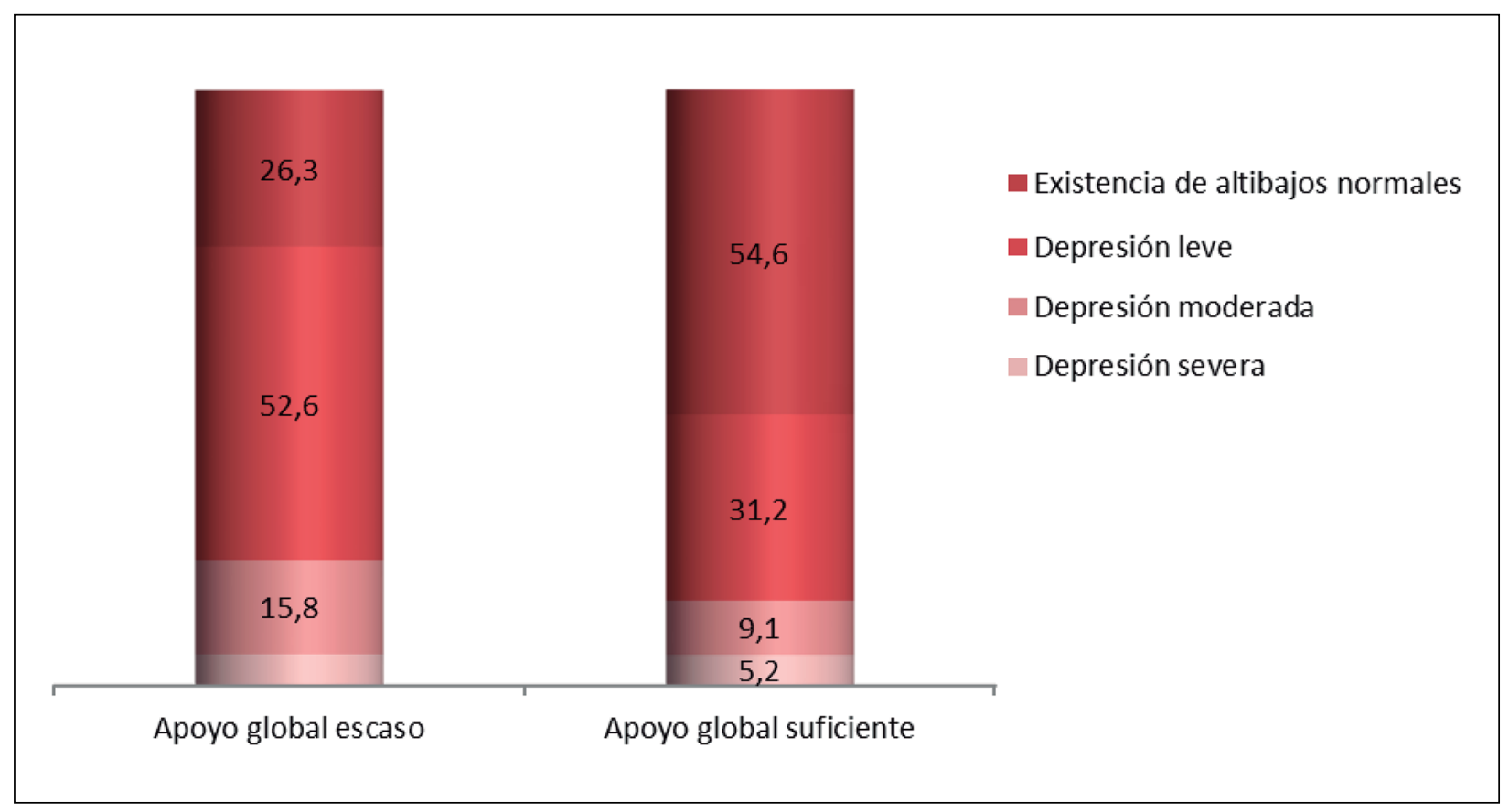

Figura 3. Depresión vinculada al apoyo social. 


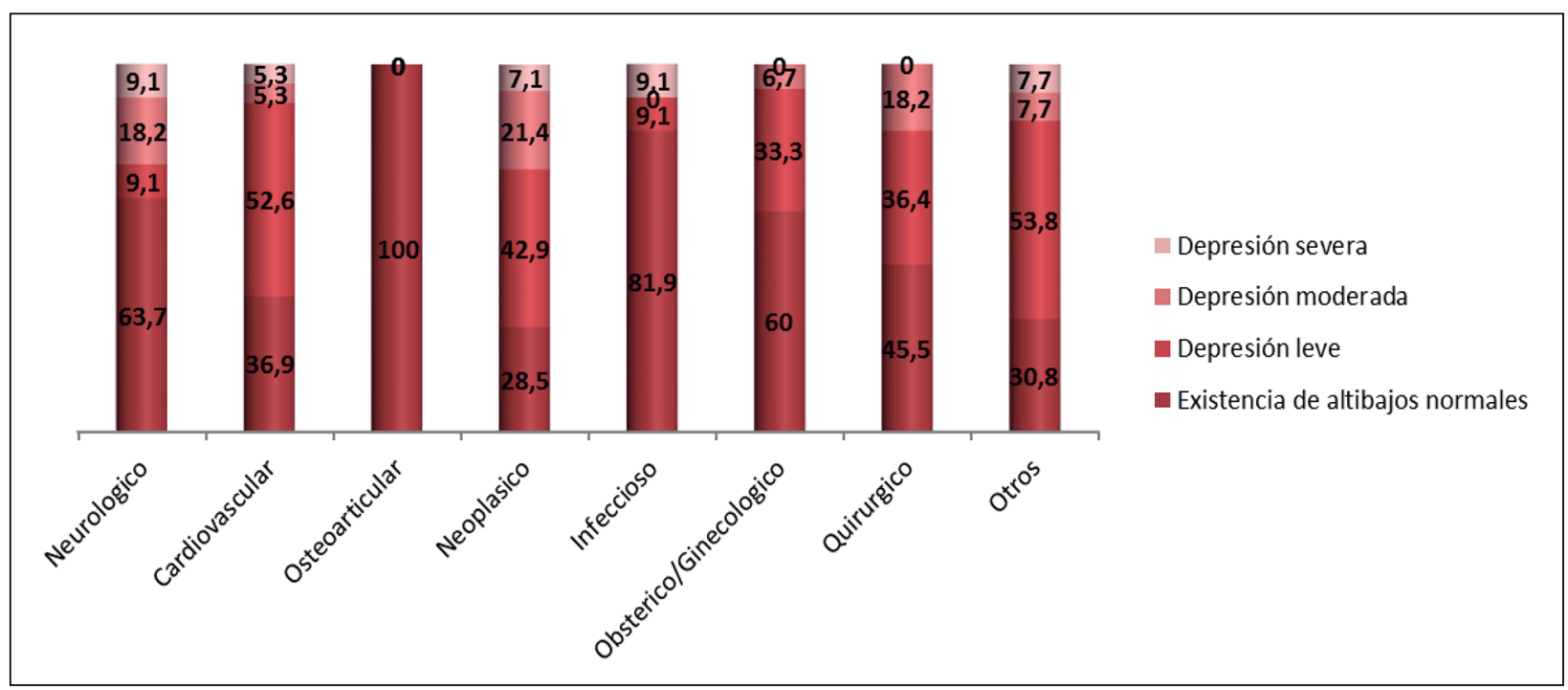

Figura 4. Depresión vinculada a patología médica.

\section{Test de significación estadística}

Con el fin de trascender el análisis descriptivo se ha aplicado la prueba $U$ de Mann-Whitney entre las diferentes variables dicotómicas relevadas (sexo, si recibe tratamiento psiquiátrico, ideas suicidas, antecedentes psiquiátricos y apoyo social) con el fin de contrastar las diferencias entre los niveles de depresión ordinales (medidos a través del test de Beck).

Para tal fin se ha establecido la hipótesis nula (Ho) que afirma la no diferencia de los valores de la escala de depresión entre los dos grupos y la hipótesis alternativa ( $\mathrm{Ha}$ ) que afirma la diferencia de los valores de la escala de depresión entre los dos grupos. Se ha asumido un nivel de confianza del 95\% $(\alpha=0,05)$.

Luego de aplicar la prueba U de Mann-Whitney para la totalidad de las variables mencionadas, concluimos que las variables que presentan un nivel de significación ( $\mathrm{p}$ valor) que permite rechazar la Ho $(\mathrm{p}<0,05)$ son: presencia de ideación suicida, antecedentes psiquiátricos y apoyo social.

Por lo tanto, es posible afirmar que existen diferencias significativas en la escala de depresión entre quienes poseen ideas de autoeliminación y quienes no, entre quienes presentan un apoyo global escaso e insuficiente (medidos a través del test de MOSS) $\mathrm{y}$, por último, entre quienes presentan antecedentes psiquiátricos y quienes no.

\section{Discusión}

La prevalencia de síntomas de depresión coincide con la reflejada en la bibliografía, confirmando su alto número en pacientes hospitalizados.

La prevalencia de ideas de autoeliminación resultó baja en contraste con nuestra impresión subjetiva.

En concordancia con los antecedentes es posible afirmar con un $95 \%$ de confianza que existen diferencias significativas en la incidencia de depresión entre quienes poseen un apoyo global escaso y quienes presentan un apoyo global suficiente (medido a través de la escala MOS).

En esta muestra la presencia o ausencia de apoyo familiar no parece ser un determinante para las depresiones graves.

Del total de pacientes entrevistados 15 presentaron depresión moderada o severa. De estos 6 fueron citados a consulta a la brevedad, 2 ya 
se encontraban en tratamiento antes del ingreso al hospital, los 7 restantes se negaron a recibir atención.

Los 5 pacientes que presentaron ideas suicidas se encontraban dentro del grupo depresión moderada-severa. De la valoración posterior se inició tratamiento en 3 de ellos, uno ya estaba en seguimiento y no se modificaron sus indicaciones, el último no concurrió.

No se había solicitado interconsulta por el equipo de psiquiatría para ninguno de los pacientes que presentaron depresión moderada y severa.

Se observa cómo diferentes estudios vinculan de una manera u otra el estado emocional afectivo y las enfermedades o padecimientos físicos. De manera tal que resulta fundamental atender el estado de ánimo de las personas, bien sea a modo de prevención de enfermedades físicas o a modo de apoyo y acompañamiento para aquellos pacientes que ya se encuentran hospitalizados. Se sugiere la creación de programas intrahospitalarios que incorporen terapias integrales que, a su vez, atiendan la dimensión emocional y afectiva de los pacientes.

\section{Limitaciones y sesgos}

Intentamos prevenir sesgos del investigador teniendo algunas precauciones metodológicas como, por ejemplo, registrar solo eventos objetivos, utilizar cuestionarios válidos y testados, entrenar a los encuestadores en el uso de instrumentos.

Se minimizaron los sesgos de selección, entrevistando al universo de pacientes internados, no limitándonos a las interconsultas solicitadas por los colegas ya que esto podría inclinar la población al subgrupo más grave. Sin embargo, se registraron 74 pérdidas en 170 pacientes, las cuales no tendríamos por qué pensar que se distribuyen de manera inhomogénea.

Podemos esperar encontrarnos con sesgos de información, ya que los datos recabados dependerán muchas veces de la memoria y subjetividad de los entrevistados ${ }^{25}$.

Debemos tener en cuenta que las preguntas 14 a 21 de la escala de depresión de Beck se pueden ver influenciadas por la presencia de enfermedad física.

Planteamos sería pertinente llevar a cabo estudios prospectivos enfocados en el paciente con escaso apoyo social y sus complicaciones.

\begin{abstract}
Resumen
Introducción: La prevalencia de depresion y ansiedad en usuarios internados en hospitales generales es muy alta, esto se asocia a mayor morbimortalidad y mayores costos en salud. El apoyo emocional resulta fundamental en todo proceso de recuperación. Método: Se realizó un estudio descriptivo y analítico sobre depresión y su vinculación con el apoyo social, estancia hospitalaria y enfermedad médica en pacientes internados en el Hospital de Clínicas Dr. Manuel Quintela Montevideo, Uruguay. Se les aplicó el test de Beck para depresión y la escala validada para soporte social Medical Outcomes Study (MOS). Se describieron características de la población estudiada. Resultados: Más de la mitad de los entrevistados presentaban algún grado de depesión. Fue posible afirmar que 73,7\% de los usuarios con apoyo social escaso presentó síntomas depresivos con un aumento de la depresión sobre todo de tipo moderada y leve. Hubo una mayor incidencia de depresión en pacientes con estancia hospitalaria prolongada, sin embargo, este dato no fue estadísticamente significativo. En cuanto a la patología médica destacamos la fuerte presencia de depresión en los pacientes oncológicos cardiovasculares $y$ quirúrgicos.
\end{abstract}

Palabras clave: Depresión, Soporte social, Enfermedad médica. 


\section{Referencias bibliográficas}

1. Hernández-Benítez CT, García-Rodríguez A, Leal-Ugarte E, Peralta-Leal V, Durán-González J. Factores ambientales relacionados a trastornos depresivos. Rev Med Inst Mex Seguro Soc 2014; 52 (5): 574-9.

2. Grau Martín A, Suñer Soler R, Abulí Picart P, Comas Casanovas P. Ansiedad y Depresión en enfermos hospitalizados y su relación con la gravedad de la enfermedad. Med Clin (Barc) 2003; 120 (10): 370-5.

3. Hernández G, Orellana G, Kimelman M, Núñez C, Ibáñez C. Trastornos de ansiedad en pacientes hospitalizados en medicina interna. Rev Med Chile 2005; 133: 895-902.

4. Martínez-Mendoza JA, Martínez-Ordaz VA, Esquivel-Molina CG, Velasco-Rodríguez VM. Prevalencia de depresión y factores de riesgo en el adulto mayor hospitalizado. Rev Med Inst Mex Seguro Soc 2007; 45 (1): 21-8.

5. DiMatteo MR, Lepper HS, Croghan TW. Depression is a Risk Factor for noncompliance with medical treatment. Arch Intern Med 2000; 160 (14): 2101-7.

6. Almada R, Miraballes R, Agrafojo S. Frecuencia de depresión en los pacientes que consultan en policlínicas de Medicina Interna de Hospital de Clínicas. Rev Psiquiatr Urug 2014; 78 (2): 11630.

7. Schwartzmann L, Caporale B, Suárez A, Sancristóbal S. Incidencia de trastornos psíquicos en pacientes con enfermedades médicas: ¿qué hacer? Rev Psiquiatría Uruguay 2003; 67: 21-38.

8. von Mühlenbrock F, Gómez R, González M, Rojas A, Vargas L, von Mühlenbrock C. Prevalencia de Depresión en pacientes mayores de 60 años hospitalizados en el Servicio de Medicina Interna del Hospital Militar de Santiago. Rev Chil NeuroPsiquiat 2011; 49 (4): 331-7.

9. Yanzón de la Torre A, Oliva N, Echevarrieta PL, Pérez BG, Caporusso GB, Titaro AJ, et al. Major depression in hospitalized Argentine general medical patients: Prevalence and risk factors. J Affect Disord 2016; 197: 36-42.

10. Agudelo D, Lucumí L, Santamaría Y. Evaluación de la depresión en pacientes hospitalizados por distintas enfermedades médicas en la ciudad de Bucaramanga. Pensamiento Psicológico 2008; 4 (10): 59-83.

11. Molina J, Uribe A, Figueroa J. Dolor, calidad de vida y estado anímico relacionados con la salud de pacientes ancianos hospitalizados. Agencia de Evaluación de Tecnologías Sanitarias de Andalucía (Aetsa), Sevilla (España). Pensamiento Psicológico 2013; 11 (1): 43-53.

12. Argimón Pallás JM, Villa JJ. Métodos de la investigación clínica y epidemiológica. Cap 4. Clasificación de los tipos de estudio. Pags. 29-31.

13. Argimón Pallás JM, Villa JJ. Métodos de la investigación clínica y epidemiológica Cap 9. Estudios descriptivos. Pags 90-100.

14. Sitio web Hospital de clínicas www.hc.edu.uy.

15. Sanz J, Vázquez C. Fiabilidad, validez y datos normativos del inventario para la depresión de beck . Psicothema [Internet] 1998; 10 (2): 303-18. Recuperado de: https://www.redalyc.org/articulo. oa?id=72710207.

16. Emmons CA, Fetting JH, Zonderman AB. A comparison of the symptoms of medical and psychiatric patients matched on the Beck Depression Inventory. Gen Hosp Psychiatry 1987; 9 (6): 398-404.

17. Beck AT, Steer RA, Carbin MG. Psychometric properties of the Beck Depression Inventory: twenty five years of evaluation. Clin Psychol Rev 1988, 8: 77-100.

18. Vega-Dienstmaier JM, Coronado-Molina O, Mazzotti G. Validez de una versión en español del Inventario de Depresión de Beck en pacientes hospitalizados de medicina general. Rev Neuropsiquiatría 2014; 77 (2): 95-103.

19. Espínola R, Carmelo E. Validación argentina del cuestionario MOS de apoyo social percibido. Universidad de Palermo 2006. http $>/ /$ hdl.handle. net/10226/428.

20. Argimón Pallás JM, Villa JJ. Métodos de investigación clínica y epidemiológica Cap 20. Diseño de cuestionarios. Pags 184-95.

21. El Senado y la Cámara de Representantes de la República Oriental del Uruguay, reunidos en Asamblea General Ley No 18.335 Pacientes 
y Usuarios de los Servicios de Salud, http:// www.parlamento.gub.uy/leyes/AccesoTextoLey. asp?Ley $=18335 \&$ Anchor.

22. Ballardo E, Rodríguez H, Rotondo T, et al. Comisión especial para ajustar el texto del proyecto del código de ética del SMU. Código de ética médica aprobado en abril de 1995. Ediciones SMU.

23. La ética médica, Normas códigos y declaraciones internacionales, ediciones SMU 1994.
24. Argimón Pallás JM, Villa JJ. Métodos de investigación clínica y epidemiológica. Cap 3. Ética e investigación. Pags 16-26.

25. The belmont report. Office of the secretary. Ethical principles and guidelines for the protection of human subjects of biomedical and behavioral research. April 18, 1979. Official site US department of Health and Human services. www.hhs.gov/ ohrp/humansubjects/guidance/belmont.html.
Correspondencia:

Alejandra Moreira R.

Rosa Guiffuni $233 \mathrm{La}$ Paz Canelones CP

Teléfono: 099086696.

E-mail: alemore@montevideo.com.uy

Pablo Fieltiz G.

E-mail: pfielitz@gmail.com 